\title{
INVASIVE LOBULAR CANCER OF THE BREAST: THE ROLE OF MRI
}

\author{
DS Raje, Senior SHO; R Bollard, Consultant, Department of Surgery \\ Royal Lancaster Infirmary
}

\section{INTRODUCTION}

Invasive lobular breast cancer (ILC) is uncommon, and affects about $10-15 \%$ of all women with breast cancer. It can occur at any age, but more commonly affects women in the 45-55 year age group. Men can also get invasive lobular breast cancer but this is very rare. These cancers often tend to be multi-focal and are also more likely to be hidden in the normal breast tissue seen on mammograms. Hence they are difficult to detect by routine screening methods, making residual disease more frequent.

ILC is characterized microscopically by similar cells forming linear invasive columns that are loosely dispersed, whereas invasive ductal carcinoma (IDC) is more typically a discrete mass. ILC frequently invades the normal tissues without invoking the vigorous desmoplastic response that usually accompanies IDC. Cells of ILC often encircle ducts, thus preserving the architecture of the ducts. These histopathologic features tend to produce more subtle imaging findings with ILC than with IDC.

An audit was conducted at RLI to review the patients with invasive lobular cancer who had further surgery due to inadequate clearance of the disease. One of the aims was to investigate the role of preoperative magnetic resonance imaging (MRI) in ILC.

\section{METHODS}

We undertook a retrospective study of 21 cases of ILC treated at the RLI. All patients had only preoperative mammograms, ultrasound and core biopsies. This data was then compared with data from centres using preoperative MRI in addition to the above investigations.

\section{RESULTS}

At RLI: Seven out of 21(33\%) patients had further surgery. Five of these had been detected at screening and two were symptomatic.

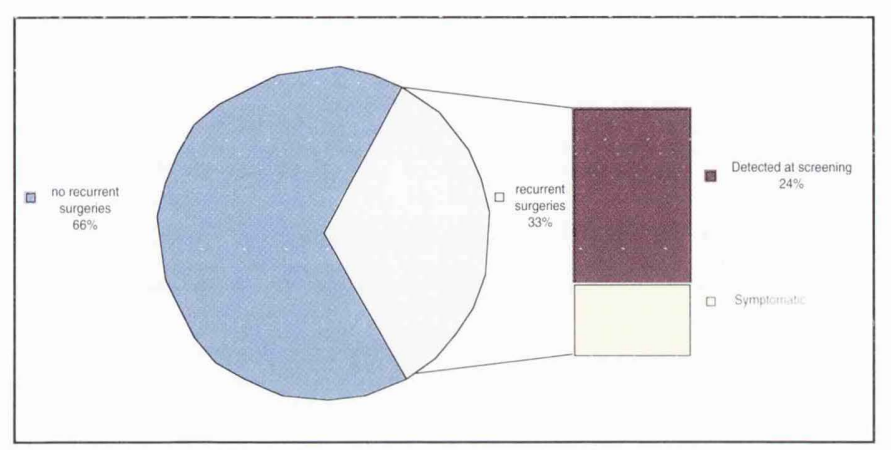

At RLI
At the centre conducting preoperative MRI ${ }^{(1)}$ : Six out of 20 patients had ILC detected in spite of normal or indeterminate mammograms and ultrasounds.

Fourteen of the 20 patients had MRI for additional information. Seven out of fourteen had significant additional information regarding tumour size. Two had contra lateral breast. involvement and one had pectoral muscle invasion.

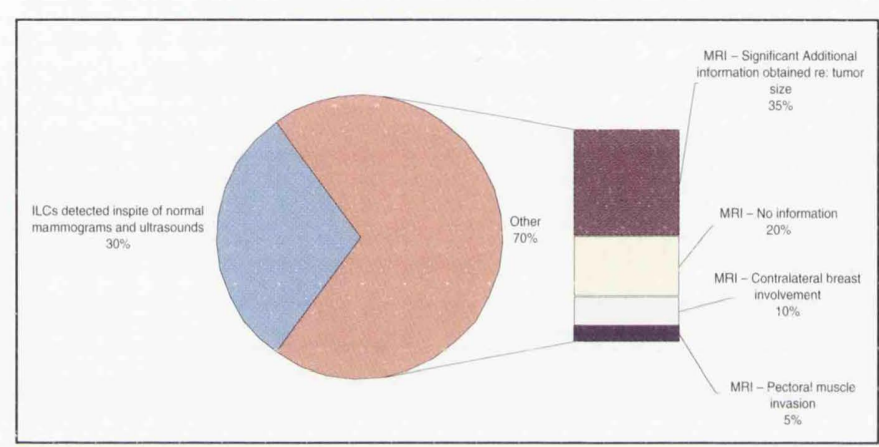

Center conducting pre-opeative MRI

Tumour correlation between MRI findings and histological extent was $(\mathrm{r}=0.967)$ as compared to $(\mathrm{r}=0.673)$ and $(\mathrm{r}=$ 0.663 ) for ultrasound and mammograms respectively.

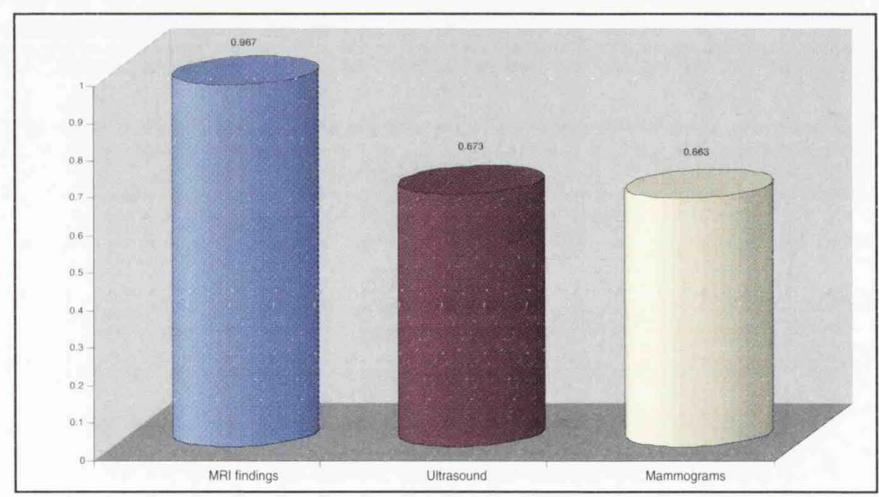

Correlation to histological extent

\section{DISCUSSION}

ILC is generally no more serious than other types of breast cancer. However, it is sometimes found in both breasts at the same time and there is also a slightly greater risk of it occurring in the opposite breast at a later date.

It can sometimes be difficult to diagnose. This is because it is less likely to present as a firm lump and is therefore not easy to feel. It is also more difficult to see on a mammogram. This is because the white dots (calcifications) that may be seen on a mammogram with other types of breast cancer are not usually formed by invasive lobular cancers.

ILC can sometimes affect more than one area within the breast. 
Contract medium enhancement (CME) MRI of the breast is a highly sensitive method for the detection of breast cancer $^{(2)}$. However, absence of contrast-medium enhancement, or delayed enhancement, does not exclude malignancy. Furthermore, some features of benign lesions were associated with contrast-medium enhancement, which lead to false positive findings, resulting in a rather low specificity for CME-MRI. When mammography and CMEMRI were used together they seemed to be complementary and a very high sensitivity (99\%) was achieved. CME-MRI was effective in revealing mammographically occult or equivocal lesions and multifocal tumours, even in dense breasts, but it was less reliable for some invasive lobular cancers. The question, therefore, is whether or not MRI can do better than mammography in assessing the size, margins, number, and locations of the cancer. In short, it probably can.

There have been claims that MRI finds additional cancers in about a third of patients (in the ipsilateral breast, not the contra lateral breast) ${ }^{(3)}$. Most centres, however, will not experience numbers this high. One of the problems is that none of the papers actually gave the size of the additional cancer, or whether it was located within $2 \mathrm{~mm}$ of the primary cancer or in another quadrant. We know that patients who have a primary cancer have additional cancers. We also know that breast cancer is in some ways like prostate cancer in that as one gets older, the likelihood of having either a non-life threatening prostate cancer or breast cancer increases exponentially. Most older women have a focus of breast cancer, but it will take many years before it grows, and most of these women are not going to succumb to it. So the fact that so many papers have documented the discovery of additional cancers without telling the significance, the size, or the location, does not really help the medical community very much. Nevertheless, the claim can still be made that MRI is more accurate for determining the size of cancers, especially in patients with dense breasts or larger breasts.

The real question, though, is whether MRI can improve survival. The answer is probably not. It may alter therapy in $11 \%-18 \%$ of cases, and that is good. Altered therapy, however, may mean that the patient is someone who would have had a lumpectomy and not a mastectomy, but the survival is going to be unchanged. So MRI is not going to change the survival, but it may alter the therapy, and in the long run that is a good role for MRI to play in selected patients

A new study shows that MRI has been shown to be effective in detecting and staging invasive lobular breast cancer, a form of breast cancer that historically has been difficult to diagnose accurately by mammography or ultrasound $^{(4)}$.

This form of cancer may be hidden within the normal breast tissue on the mammogram and therefore cannot be easily seen. MRI appears to be much more informative.

One study evaluated the use of MRI as an extension of existing breast imaging technologies ${ }^{(5)}$. Fifty-four patients with clinically and/or mammographically suspicious breast lesions were evaluated with MRI in addition to a clinical exam, mammography, and cytology.

MRI had a sensitivity of $91 \%$ and a specificity of $67 \%$. MRI detected 30 of 33 malignancies and had seven false positives. It was found to be useful in women with dense breasts and especially for patients with clinical evidence of breast carcinoma that could not be detected with current diagnostic procedures.
MRI of the breast has a high sensitivity but low specificity. Conventional mammography can be more sensitive in detecting in situ carcinomas and also invasive carcinomas. A negative MRI exam cannot exclude malignancy. This means that one still cannot completely rely on the MRI diagnosis for women with mammographically detected lesions that show no enhancement on the MRI examination. MRI is complimentary to mammography.

Contrast-enhanced MRI has been shown to have value in the diagnostic work-up of women who present with mammogram or clinical abnormalities. In addition, it has been demonstrated that MRI can detect mammogram occult multifocal cancer in patients who present with unifocal disease. Advances in risk stratification and limitations in mammography have stimulated interest in the use of MRI to screen high-risk women for cancer. Several studies of MRI high-risk screening are ongoing. Preliminary results are encouraging.

Despite the success of mammography screening, mammography does have limitations. Perhaps its most significant limitation is the difficulty in detecting masses within radiographically dense breasts. In addition, cancers can be missed by mammography. Retrospective studies of breast cancer in which prior mammograms were read as negative showed that the cancer was visible in retrospect in approximately one-third of the cases. In addition, the relatively low specificity of mammography leads to many breast biopsies that reveal benign tissue.

The detection of breast cancer with MRI is based on the fact that nearly $100 \%$ of invasive cancers will enhance with the administration of IV gadolinium ${ }^{(6)}$. This is probably on the basis of invasive tumor angiogenesis. The true false negative rate, however, is probably not known. For in situ cancers, MRI detects only $40-100 \%$ of lesions. The variability of sensitivity reflects the inclusion of some tumors with microinvasion, some series with small numbers of reported cases, and variable histology.

Breast MRI may be superior to mammography and ultrasound for the screening of women at high risk for hereditary breast cancer. Mammographically, often no focal mass or clustered microcalcifications are evident, particularly in dense parenchyma. As a result, ILC tends to be larger than IDC at diagnosis, with an average size of $29 \mathrm{~mm}$ compared with $23 \mathrm{~mm}$ for IDC in one series. Hilleren et al retrospectively reviewed 137 cases of ILC and found that 22 (16\%) appeared mammographically occult or benign. Of those visible mammographically, more than one third were seen as vague asymmetries, poorly defined opacities, or architectural distortions. In two series, ILC was better visualized in the craniocaudal view. Calcifications are often the earliest manifestation of ductal carcinomas but are uncommon in ILC. Calcifications were seen in only three $(2 \%)$ of 137 ILCs in the series of Hilleren $e \mathrm{al}^{77}$.

Because of the limitations of mammography in detecting ILC, other modalities, such as sonography and MRI, are being used in evaluating clinically suspicious findings and known cancers to assess the extent of disease. In a recent and much larger series by Butler et $a l^{(8)} 208$ cases of ILC were reviewed. Of the 208 cases of ILC, 81 (39\%) were mammographically subtle or invisible, and 71 (88\%) of these 81 ILC were depicted sonographically. Average tumour size was $2.5 \mathrm{~cm}$. The most common sonographic appearance was a heterogeneous, hypoechoic mass with angular or ill-defined margins and posterior acoustic shadowing, which was seen in $49(69 \%)$ of 71 sonographically visible cases. In another 12 
(17\%), focal shadowing without a discrete mass was revealed, and in $10(14 \%)$, the tumour appeared as a lobulated and well circumscribed.

As stated previously, there is considerable limitation in the depiction of lobular carcinoma at mammography, since it often does not manifest as a definite mass or as microcalcifications, and it can appear benign sonographically. As such, contrast-enhanced MRI has been increasingly proposed as an adjunctive modality for depiction of ILC as well as of IDC, and it is particularly useful for defining the extent of disease within the breast prior to definitive treatment. Weinstein et $a l^{(8)}$ performed preoperative MR imaging in 18 patients with ILC and found that in 16 (89\%), the extent of disease identified at MRI correlated with that identified at pathologic examination. Further, the extent of tumour was correctly depicted with only MR imaging in 7 (39\%) of 18 cases, with both mammography and MR imaging in nine $(50 \%)$ of 18 cases, and with neither in one $(6 \%)$ of 18 cases. Of the eight patients with more extensive tumour on the basis of MR imaging findings, surgical treatment was altered in all eight $(100 \%)$ patients.

Preoperative assessment with MR imaging may be particularly important for ILC, since the rate of positive margins at initial excision is higher than it is for IDC. Follow-up breast cancer screening using MRI can track and catch some of the smallest, early breast cancers in high-risk women while they are most susceptible to treatment. A new study $^{(9)}$ shows follow-up MRI can detect curable early breast cancers that cannot be found by mammography or physical examination.

Researchers say that a study by Liberman et $a l^{(10)}$, is the first to examine 'probably benign' findings from MRI breast cancer screening among women at high risk of developing breast cancer due to a family history of the disease or previous breast cancers.

Although breast cancer screening using MRI is very good at picking up the tiniest abnormalities in the breast, it can also pick up benign or normal growths in breast tissue. Researchers say MRI screening is less accurate at determining which of these growths may be cancerous, and the outcome of probably benign growths detected by MRI has not been studied until now.

\section{TRACKING EARLY BREAST CANCERS WITH MRI}

In the Liberman study, researchers followed 367 women at high risk of developing breast cancer who had normal mammograms and were referred for further screening using MRI.

Follow-up breast cancer screening using MRI is frequently recommended for tracking growths or abnormalities in the breast that doctors believe are probably noncancerous or benign. Researchers say breast cancer screening has several advantages over biopsy in this regard because it is noninvasive, less expensive, and causes less anxiety for the patient than biopsies, which require removal of breast tissue with a needle for further testing.

Breast cancer screening using MRI found "probably benign' growths in 89 of these women (24\%). Follow-up MRI screening was performed for $79 \%$ of these women after an average of 11 months. Most of the women who were referred for follow-up MRI had multiple growths, usually in both breasts.
Twenty of the 89 women subsequently had a biopsy after follow-up MRI due to progression of the growth. Breast cancer was found in nine women. This means that approximately $10 \%$ of the women initially diagnosed with probably benign growths had early breast cancer.

\section{CONCLUSIONS}

Underestimated tumour size was the main reason for further surgery in ILC at RLI.

Mammograms and ultrasound can fail to detect and underestimate tumour size in ILC.

Contrast-enhanced MRI is more sensitive in detecting and providing additional information in ILC.

Conventional mammography can be more sensitive in detecting in situ carcinomas and also invasive carcinomas. A negative MRI exam cannot exclude malignancy. This means that one still cannot completely rely on the MRI diagnosis for women with mammographically detected lesions that show no enhancement on the MRI examination. MRI is complimentary to mammography.

Breast MRI may be superior to mammography and ultrasound for the screening of women at high risk for hereditary breast cancer.

MRI is not going to change the survival, but it may alter the therapy, and in the long run that is a good role for MRI to play in selected patients of ILC.

\section{REFERENCES}

1 K.Munot, B.Dall, R.Achuthan, G.Parkin, S.Law, K.Horgan. Role of MRI in diagnosis and single staged surgical resection of ILC of breast. Br J Surg 2002;89:1296-1301

2 Kneeshaw PJ, Turnbull LW, Smith A, Drew PJ. Dynamic contrast enhanced magnetic resonance imaging aids the surgical management of invasive lobular breast cancer. Eur J Surg Oncol 2003;29(1):32-7

3 Moon WK, Noh D-Y, Im J-G. Multifocal, multicentric, and contralateral breast cancers: bilateral whole-breast US in the preoperative evaluation of patients. Radiology 2002;224(2):569-576

4 Bone B, Aspelin P, Bronge L, Isberg B, Perbeck L, Veress B. Sensitivity and specificity of MR mammography with histopathological correlation in 250 breasts. Acta Radiologica. 1996;37(2):208-13

5 Obdeijn I, Kuijpers T, Dijk P et al. MR lesion detection in a breast cancer population. J Magn Reson Imaging 1996;6:8498547

6 Kuhl CK et al. Dynamic gadolinium enhanced breast MR: are signal intensity time course data useful for diagnosis? Radiology 1999;211:101

7 Hilleren DJ, Andersson IT, Lindholm K, Linnell FS. Invasive lobular carcinoma: mammographic findings in a 10year experience. Radiology 1991;178:149-154

8 Weinstein SP, Orel SG, Heller R et al. MR imaging of the breast in patients with invasive lobular carcinoma. Am J Roentgenol 2001;176:399-406.

9 Kuhl CK.. High-risk screening: multi-modality surveillance of women at high risk for breast cancer (proven or suspected carriers of a breast cancer susceptibility gene). J Exp Clin Cancer Res 2002;21(3 Suppl):103-6

10 Liberman et al. MRI Beneficial for Assessing "Probably Benign" Breast Cancer Lesions in High-Risk Women, Cancer. 2003 Jul 15;98(2):377-88 